\section{Sur l'allure asymptotique des potentiels de chaleur et de} l'intégrale de Fourier-Poisson

par M. KRZYŻańs.KI (Kraków)

\section{Introduction}

1. Il est bien connu que, la fonction $p(s)$ étant mesurable (au sens de Lebesgue) et bornée pour $-\infty<s<+\infty$, l'intégrale de Fourier-Poisson

$$
I(x, t)=\frac{1}{2 \sqrt{ } \pi t} \int_{-\infty}^{+\infty} \varphi(s) \exp \left[-\frac{(x-s)^{2}}{4 \cdot t}\right] d s
$$

constitue, pour $t>0$, une solution de l'équation de la chaleur

$$
u_{x x}^{\prime \prime}=u_{t}^{\prime}
$$

et qu'elle satisfait à la condition initiale

$$
\lim _{\substack{x \rightarrow x_{0} \\ t \rightarrow 0}} I(x, t)=\varphi\left(x_{0}\right)
$$

toutes les fois que la fonction $\varphi(s)$ est continue an point $\left.s=x_{0}{ }^{1}\right)$. Si la fonction $\varphi(s)$ est continue et bornée pour $-\infty<s<+\infty$, la fonction $I(x, 1)$. est continue pour $t \geqslant 0$ (à condition d'admettre qu'aux points $(x, 0)$ de l'axe $t=0$ elle prend la valeur $\lim I(\bar{x}, t))$ et elle satisfait à la condition initiale $I(x, 0)=\varphi(x)$ $\underset{\substack{\vec{x} \rightarrow 0 \\ t \rightarrow 0}}{x \rightarrow 0}$

Si la fonction $\varphi(s)$ est mesurable et bornée pour $s \geqslant 0$, la fonction

$$
u(x, t)=\frac{1}{2 \sqrt{\pi t}} \int_{0}^{\infty} \phi(s)\left\{\exp \left[-\frac{(x-s)^{2}}{4 t}\right]-\exp \left[-\frac{(x+s)^{2}}{4 t}\right]\right\} d s
$$

est continue et satisfait à l'équation (1) pour $t>0$. Cette fonction se comporte de même que l'intégrale $I(x, t)$ pour $x \rightarrow x_{0}, t \rightarrow 0$, si $x_{0}>0$. Elle satisfait en outre à la condition aux limites $u(0, t)=0$ pour $t>0$. Si

1) Voir par exemple [1], chap. XXIX, p. 294, [3], p. 220-226. La fonction $\varphi(s)$ étant bornée, on n'introduit pas de difficultés dans la démonstration de si l'on admet qu'elle est intégrable au sens de Lebesgue. la fonction $\varphi(s)$ est continue et bornée pour $s \geqslant 0$ et s'annule pour $s=0$, la fonction $u(x, t)$ est continue pour $x \geqslant 0, t \geqslant 0$ et constitue une solution du premier problème de Fourier pour l'équation (1), avec la condition initiale

$$
u(x, 0)=\varphi(x) \quad \text { pour } \quad x \geqslant 0
$$

et la condition aux limites

$$
u(0, t)=0 \quad \text { pour } \quad t \geqslant 0 .
$$

2. Pour résoudre le problème de Fourier avec la condition initiale (3) et la condition aux limites

$$
u(0, t)=\varphi(t) \quad \text { pour } \quad t \geqslant 0,
$$

$\psi(t)$ étant une fonction continue pour $t \geqslant 0$ et s'annulant pour $t=0$ on introduit une intégrale analogue aux potentiels (newtonien, logarith mique) de double couche, à savoir

$$
J_{1}(x, t)=\frac{1}{2 \sqrt{\pi}} \int_{0}^{t} \frac{x}{(t-\tau)^{3 / 2}} \exp \left[-\frac{x^{2}}{4(t-\tau)}\right] \psi(\tau) d \tau
$$

nous appelerons cette intégrale - potentiel de chaleur de seconde espèce. Elle est continue pour $x \neq 0, t \geqslant 0$ et satisfait à l'équation (1) pour $x \neq 0$, $t>0$. Il est évident que l'on a

$$
J_{1}(0, t)=0,
$$

tandis qu'on démontre que ([1], p. 306 et [3], p. 471)

$$
\lim _{\substack{x \rightarrow 0 \\ t \rightarrow t_{0}}} J_{1}(x, t)(\operatorname{sgn} x)=\psi\left(t_{0}\right) \quad \text { pour } \quad t_{0}>0 .
$$

Les formules (6) et (7) mettent en évidence l'analogie entre l'intégrale $J_{1}(x, t)$ et les potentiels de double couche. Il résulte de la formule (7) que si $\varphi(0)=\psi(0)=0$, la solution du premier problème de Fourier pour l'équation (1) avec les conditions (3) et (5) est donnée par la formule

(8) $\quad u(x, t)=\frac{1}{2 \sqrt{\pi t}} \int_{0}^{\infty} \varphi(s)\left\{\exp \left[-\frac{(x-s)^{2}}{4 t}\right]-\exp \left[-\frac{(x+s)^{2}}{4 t}\right]\right\} d s+$

$$
+\frac{1}{2 \sqrt{\pi}} \int_{0}^{t} \frac{x}{(t-\tau)^{3 / 2}} \exp \left[-\frac{x^{2}}{4(t-\tau)}\right] \psi(\tau) d \tau
$$


3. L'intégrale

$$
J_{0}(x, t)=\frac{1}{2 \sqrt{\pi}} \int_{0}^{t} \frac{\psi(\tau)}{(t-\tau)^{1 / 2}} \exp \left[-\frac{x^{2}}{4(t-\tau)}\right] d \tau
$$

que nous appelerons potentiel de chaleur de première espèce, est analogue au potentiel de simple conche. Elle est continue pour $t \geqslant 0$ et satisfait à l'équation (1) pour $t>0, x \neq 0$. Flle permet de résoudre le second problème de Fourier qui consiste à rechercher une fonction $u(x, t)$ continue pour $x \geqslant 0, t \geqslant 0$, admettant une derivée $u_{x}^{\prime}$ continue pour $x \geqslant 0, t>0$, satisfaisant à l'équation (1) pour $x>0, t>0$, à la condition initiale (3) et à la condition anx limites

$$
u_{x}^{\prime}(0, t)=\psi(t) \text { pour } \quad t \geqslant 0 .
$$

On suppose, cette fois, que $\psi(t)$ est une fonction continue pour $t \geqslant 0$ et $\varphi(x)$ une fonction continue pour $x \geqslant 0$, admettant une dérivée continue au moins dans un intervalle $\langle 0, \gamma\rangle$, où $\gamma$ est un nombre positif. On suppose en outre que $\varphi^{\prime}(0)=\psi(0)=0$. La solution du problème qui vient d'être posé est donnée par la formule suivante

$$
\begin{aligned}
u(x, t)= & \frac{1}{2 \sqrt{\pi t}} \int_{0}^{\infty} \varphi(s)\left\{\exp \left[-\frac{(x-s)^{2}}{4 t}\right]+\exp \left[-\frac{(x+s)^{2}}{4 t}\right]\right\} d s- \\
& -\frac{1}{\sqrt{\pi}} \int_{0}^{t} \frac{\psi(\tau)}{(t-\tau)^{1 / 2}} \exp \left[-\frac{x^{2}}{4(t-\tau)}\right] d \tau .
\end{aligned}
$$

Remarque I. Si la fonction $\varphi(s)$ est mesurable et bornée pour $s \geqslant 0$, sans être partout continue, les formules (2), (8) et (10) déterminent les solutions généralisées des problèmes correspondants, énoncés aux $n^{\text {os }} 2$ et 3 ; la condition initiale (3) est satisfaite aux points de continuité de la fonction $\varphi(x)$.

Remarque II. La fonction $\varphi(x)$ ettant continue pour $x \geqslant 0$, les solutions des problèmes qu'on vient d'énoncer aux $\mathrm{n}^{\text {os }} 2-3$ sont uniques dans la classe des fonctions bornées, et même dans la classe $\boldsymbol{E}_{8}$ des fonctions $u(x, t)$ qui satisfont à une inégalité de la forme $|u(x, t)| \leqslant M e^{K x^{2}}$, $M$ et $K$ étant des constantes (qui dépendent en général de la fonction $u(x, t))$. Les formules (2), (8) et (10) subsistent, si la fonction $q(x)$ appartient à la classe $E_{2}$, e'est-à-dire si l'on a $|\varphi(x)| \leqslant M e^{K x^{2}}$.

4. Dans la présente note nous allons étudier l'allure des intégrales $I(x, t), J_{0}(x, t)$ et $J_{1}(x, t)$ lorsque la variable $t$ tend vers l'infini. On pourra étudier ensuite l'allure pour $t \rightarrow \infty$ des solutions des problèmes de Fourier qui viennent d'être énoncés. Ces recherches se prêtent à des applications physiques grâse à l'interprétation de ces problèmes dans la théorie de la propagation de la chaleur.
Étude de l'allure de l'intégrale de Fourier-Poisson pour $t \rightarrow \infty$

5. Il est évident que si la fonction $\varphi(s)$ s'annule en dehors d'un intervalle borné $\langle a, b\rangle$ et reste mesurable et bornée dans l'intervalle $\langle a, b\rangle$ lui-même, l'intégrale $I(x, t)$ tend vers zéro pour $t \rightarrow \infty$. L'exemple qui va suivre montre qu'il n'en est pas ainsi en général. Nous allons choisir la fonction $\varphi(s)$ mesurable et bornée de sorte que l'intégrale $I(x, t)$ n'ait pas de limite pour $t \rightarrow \infty$.

Considérons l'ensemble $E$, constitué par la suite d'intervalles $\left\langle n^{n}, h_{n} \eta^{n}\right\rangle$, où $h_{n}=\frac{1}{2}\left[(-1)^{n}+1\right]+2$. Soit

$$
\varphi(s)= \begin{cases}1 & \text { aux points de l'ensemble } E \\ 0 & \text { en dehors de l'ensemble } E\end{cases}
$$

On a alors

$$
I(0, t)=\frac{1}{\sqrt{\pi}} \sum_{n=1}^{\infty} \int_{n^{n} / 2 / \bar{t}}^{h_{n} n^{n} / 2 \sqrt{t}} e^{-\sigma^{2}} d \sigma .
$$

En particulier, $p$ étant un nombre entier positif, on a

$$
I\left(0, p^{2 p}\right)=\frac{1}{\sqrt{\pi}}\left[A_{p p}+\sum_{n=1}^{p-1} A_{n p}+\sum_{n=p+1}^{\infty} A_{n p}\right],
$$

où l'on a posé

$$
A_{n p}=\int_{n^{n} / 2 p^{p}}^{h_{n} n^{n / 2 p^{p}}} e^{-\sigma^{2}} d \sigma
$$

Comme

$$
A_{n p}<\int_{n^{n} / 2 p^{p}}^{3 n n / 2 p p^{p}} e^{-\sigma^{2}} d \sigma<\int_{n^{n} / 2 p^{p}}^{3 n n / 2 p^{p}} d \sigma=n^{n} / p^{p}
$$

on a

$$
\sum_{n=1}^{p-1} A_{n p}<\frac{(p-2)^{p-1}}{p^{p}}+\frac{(p-1)^{p-1}}{p^{p}}<2 \frac{(p-1)^{p-1}}{p^{p}}=\frac{2}{p}\left(\frac{p-1}{p}\right)^{p-1},
$$

d'où il s'ensuit

D'autre part

$$
\lim _{p \rightarrow \infty} \sum_{n=1}^{p-1} A_{n p}=0
$$

$$
\sum_{n=p+1}^{\infty} A_{n p}<\int_{(p+1)^{p+1} / 2 p^{p}}^{\infty} e^{-\sigma^{2}} d \sigma<\int_{(p+1) / 2}^{\infty} e^{-\sigma^{2}} d \sigma,
$$


on a done aussi

$$
\lim _{p \rightarrow \infty} \sum_{n=p+1}^{\infty} A_{n p}=0 .
$$

Il en résulte que si $p$ est assez grand, la contribution des sommes

$$
\sum_{n=1}^{p-1} A_{n p} \quad \text { et } \quad \sum_{n=1}^{\infty} A_{n p}
$$

au second membre de (11) est négligeable, de sorte qu'il suffit d'examiner l'allure de l'intégrale

$$
A_{p p}=\int_{1 / 2}^{h_{p / 2}} e^{-\sigma^{2}} d \sigma .
$$

Or cette intégrale n'a pas de limite pour $p \rightarrow \infty$, il en est donc de même de l'intégrale $I\left(0, p^{2 p}\right)$; il en résulte que l'intégrale $I(0, t)$ n'a pas de limite pour $t \rightarrow \infty$.

On voit aisément de quelle manière il faut modifier la fonction $\varphi(s)$, pour obtenir une fonction continue et telle que l'intégrale $I(x, t)$ correspondante jonisse de la même propriété que celle qui vient d'être construite.

6. Nous allons pourtant démontrer le théorème suivant:

THÉoRìme. Soit $\varphi(s)$ une fonction mesurable (au sens de Lebesgue) et bornée pour $-\infty<s<+\infty$. On suppose en outre l'existenve des limites

$$
\lim _{s \rightarrow+\infty} \varphi(s)=l^{+} \quad \text { et } \quad \lim _{s \rightarrow-\infty} p(s)=l^{-}
$$

Alors, pour toute valeur de $x$, on a

$$
\lim _{t \rightarrow \infty} I(x, t)=\frac{1}{2}\left(l^{+}+l^{-}\right) .
$$

Démonstration. On ramène l'intégrale $I(x, t)$ à la forme

$$
I(x, t)=\frac{1}{\sqrt{\pi}} \int_{-\infty}^{+\infty} e^{-\sigma^{2}} p(x+2 \sigma \sqrt{t}) d \sigma
$$

(forme de Fourier) en posant $s=x+2 \sigma \sqrt{t}$. Il existe un nombre positif $M$, tel que $|\varphi(s)| \leqslant M$ pour $-\infty<s<+\infty$. Choisissons le nombre $N$ de sorte que l'on ait

Alors on a

$$
\int_{|\sigma|>N} e^{-\sigma^{2}} d \sigma<\frac{\varepsilon}{3 M}
$$

$$
\left|\int_{|\sigma|>N} e^{-\sigma^{2}} \varphi(x+2 \sigma \sqrt{t}) d \sigma\right|<\varepsilon / 3 .
$$

D'autre part, la fonction $\varphi$ étant bornée, l'intégrale

$$
\int_{-N}^{N} e^{-\sigma^{2}} \varphi(x+2 \sigma \sqrt{t}) d \sigma=\int_{-N}^{0} e^{-\sigma^{2}} \varphi(x+2 \sigma \sqrt{t}) d \sigma+\int_{0}^{N} e^{-\sigma^{2}} \varphi(x+2 \sigma \sqrt{t}) d \sigma
$$

tend, d'après le théorème de Lebesgue (voir p. ex. [2], p. 44), vers la limite

$$
l_{1} \int_{-N}^{0} e^{-\sigma^{2}} d \sigma+l_{2} \int_{0}^{N} e^{-\sigma^{2}} d \sigma=\frac{V^{\prime} \pi}{2}\left(l_{1}+l_{2}\right)-\left[l_{1} \int_{-\infty}^{-N} e^{-\sigma^{2}} d \sigma+l_{2} \int_{\dot{N}}^{\infty} e^{-\sigma^{2}} d \sigma\right],
$$

pour $t \rightarrow \infty$. Il résulte de (13) qu'on peut choisir le nombre $T$ de sorte que l'on ait

$$
\left|\int_{-N}^{N} e^{-\sigma^{2}} \varphi(x+2 \sigma \sqrt{t}) d \sigma-\frac{\sqrt{\pi}}{2}\left(l_{1}+l_{2}\right)\right|<\frac{2}{3} \varepsilon
$$

pour $t>T$. On a donc, d'après (14) et (15),

pour $t>T$.

$$
\left|I(x, t)-\frac{1}{2}\left(l_{1}+l_{2}\right)\right|<\varepsilon
$$

Le nombre positif $\varepsilon$ ayant été choisi d'une manière arbitraire, on a done

$$
\lim _{t \rightarrow \infty} I(x, t)=\frac{1}{2}\left(l_{1}+l_{2}\right)
$$

CoRollaIRE. Soit $\varphi(x)$ une fonction mesurable et bornée pour $x \geqslant 0$, admettant la limite

$$
\lim _{x \rightarrow \infty} \varphi(x)=l .
$$

Si $u_{1}(x, t)$ est la solution du premier problème de Fourier, avec les conditions (3) et (4), définie par la formule (2), on a

$$
\lim _{t \rightarrow \infty} u_{1}(x, t)=0 \text {. }
$$

Si $u_{2}(x, t)$ est la solution du second problème de Fourier, avec la condition initiale (3) et la condition aux limites

$$
u_{x}^{\prime}(0, t)=0 \quad(\text { pour } t>0),
$$

définie par la formule (10) (où l'on a, cette fois, $\psi(\tau) \equiv 0$, de sorte que la seconde intÉgrale est nulle), alors

$$
\lim _{t \rightarrow \infty} u_{2}(x, t)=l \text {. }
$$

En effet, on a d'après la formule (2), resp. la formule (10),

$$
u_{k}(x, t)=\frac{1}{2 \sqrt{\pi t}} \int_{\infty}^{+\infty} \Phi_{k}(s) \exp \left[-\frac{(x-s)^{2}}{4 t}\right] d s \quad(k=1,2),
$$


où

$$
\begin{aligned}
& \Phi_{1}^{\prime}(s)=\left\{\begin{array}{ccc}
\varphi(s) & \text { pour } & s \geqslant 0, \\
-\phi(-s) & \text { pour } & s<0
\end{array}\right. \\
& \Phi_{2}(s)=\left\{\begin{array}{ccc}
\varphi(s) & \text { pour } & s \geqslant 0, \\
\varphi(-s) & \text { pour } & s<0
\end{array}\right.
\end{aligned}
$$

et, par conséquent,

$\lim _{s \rightarrow+\infty} \Phi_{1}(s)=\lim _{s \rightarrow+\infty} \Phi_{2}(s)=l, \quad \lim _{s \rightarrow-\infty} \Phi_{1}(s)=-l, \quad \lim _{s \rightarrow \rightarrow \infty} \Phi_{2}(s)=l$.

7. Quant à la fonction $u(x, t)$, représentée par la formule (2), on peut démontrer un théorème plus général.

THÉoR̀̀me. Soit $u(x, t)$ la fonction, représentée pour $x \geqslant 0, t>0$ par la formule $(2), \varphi(s)$ étant une fonction mesurable et bornée pour $s \geqslant 0$. On a

$$
\lim _{t \rightarrow \infty} u(x, t)=0
$$

pour toute valeur positive de $x$, cette convergence étant uniforme par rapport $\grave{a} x$ pour $0 \leqslant x \leqslant R$, ò̀ $R$ est un nombre positif arbitraire.

Démonstration. Soit

$$
M=\sup _{s \geq 0}|q(s)| .
$$

Observons que l'on a $|x-s| \leqslant x+s$ pour $x \geqslant 0, s \geqslant 0$, done de la formule (2) résulte l'inégalité

$$
|u(x, t)| \leqslant \frac{M}{2 \sqrt{\pi t}} \int_{-\infty}^{+\infty}\left\{\exp \left[-\frac{(x-s)^{2}}{4 t}\right]-\exp \left[-\frac{(x+s)^{2}}{4 t}\right]\right\} d s,
$$

c'est-à-dire

$$
|u(x, t)| \leqslant \frac{M}{\sqrt{\pi}} \int_{-x / 2 \sqrt{t}}^{x / 2 \sqrt{t}} e^{-\sigma^{2}} d \sigma
$$

Or le second membre de cette inégalité tend vers zéro pour $t \rightarrow \infty$ uniformément par rapport à $x$ poưr $0 \leqslant x \leqslant R$, où $R$ est un nombre positif arbitraire. II en est done de même de la fonction $u(x, t)$.

\section{Étude de l'intégrale $\mho_{1}(x, \imath)$}

8. Nous allonis démontrer un théorème analogue, relatif au potentiel calorifique $J_{1}(x, t)$. $\nabla u$ le sens physique de la fonction $\psi(t)$, qui figure sous le signe de cette intégrale (voir $\mathrm{n}^{\circ} 2$ ), nous bornerons notre étude au cas où la fonction $\psi(t)$ est continue pour $t \geqslant 0$.
THÉORÈME. Si la fonotion $\psi(t)$ est continue pour $t \geqslant 0$ et s'il existe la limite

alors on $a$

$$
\lim _{t \rightarrow \infty} \psi(t)=L,
$$

$$
\lim _{t \rightarrow \infty} J_{1}(x, t)=L
$$

pour toule valeur positive (fixée) de la variable $x$.

Démonstration. La fonction $\psi(t)$ étant continue pour $t \geqslant 0$ et ayant une limite pour $t \rightarrow \infty$, elle est bornée pour $t \geqslant 0$, de sorte qu'il existe un nombre positif $M$ tel que l'on ait $|\psi(t)| \leqslant M$ pour $t \geqslant 0$. Il est évident que $|L| \leqslant M$. Posons

On a

$$
V(x, t ; \xi, \tau)=\frac{x-\xi}{(t-\tau)^{3 / 2}} \exp \left[-\frac{(x-\xi)^{2}}{4 \cdot(t-\tau)}\right]
$$

or

$$
J_{1}(x, t)=\frac{1}{2 \sqrt{\pi}} \int_{0}^{t} V(x, t ; 0, \tau) \psi(\tau) d \tau
$$

$$
\begin{gathered}
\frac{1}{2 \sqrt{ } \pi} \int_{0}^{t} V(x, t ; 0, \tau) d \tau=\frac{2}{\sqrt{\pi}} \int_{x / 2 \sqrt{t}}^{\infty} e^{-\sigma^{2}} d \sigma \\
=\frac{2}{\sqrt{\pi}}\left[\int_{0}^{\infty} e^{-\sigma^{2}} d \sigma-\int_{0}^{x / 2 \sqrt{t}} e^{-\sigma^{2}} d c\right]=1-\frac{2}{\sqrt{\pi}} \int_{0}^{x / 2 \sqrt{t}} e^{-\sigma^{2}} d \sigma,
\end{gathered}
$$

et, par suite,

$$
\text { (18) } \quad J_{1}(x, t)-L=\frac{1}{2 \sqrt{\pi}} \int_{0}^{t} V(x, t ; 0, \tau)[\psi(\tau)-L] d \tau-\frac{2 L}{\sqrt{\pi}} \int_{0}^{x / 2 \sqrt{t}} e^{-\sigma^{2}} d \sigma
$$

Le second terme du second membre de (18) tend vers zéro pour $t \rightarrow \infty$. Il nous reste à démontrer qu'il en est de même du premier terme. A cet effet, nous choisissons le nombre $T_{0}$ de façon que l'on ait

$$
|\psi(t)-L|<\varepsilon / 2 \quad \text { pour } \quad t>T_{0} .
$$

Soit $t>T_{0} ;$ on a

$$
\frac{1}{2 \sqrt{\pi}} \int_{0}^{t} \nabla(x, t ; 0, \tau)[\psi(\tau)-L] d \tau
$$

$$
=\frac{1}{2 \sqrt{\pi}} \int_{0}^{T_{0}} V(x, t ; 0, \tau)[\psi(\tau)-L] d \tau+\frac{1}{2 \sqrt{\pi}} \int_{T_{0}}^{t} V(x, t ; 0, \tau)[\psi(\tau)-L] d \tau .
$$


D'après $(19)$ on a

(20) $\left|\frac{1}{2 \sqrt{\pi}} \int_{T_{0}}^{t} V(x, t ; 0, \tau)[\psi(\tau)-L] \mathrm{d} \tau\right| \leqslant \frac{\varepsilon}{\sqrt{\pi}} \int_{0}^{\infty} e^{-\sigma^{2}} d \sigma=\frac{\varepsilon}{2}$.

D'autre part,

$$
\left|\frac{1}{2 \sqrt{\pi}} \int_{0}^{T_{0}} V(x, t ; 0, \tau)[\psi(\tau)-L] d \tau\right| \leqslant \frac{M}{\sqrt{\pi}} \int_{0}^{T_{0}} V(x, t ; 0, \tau) d \tau,
$$

et, par suite, .

$$
\left|\frac{1}{2 \sqrt{\pi}} \int_{0}^{T_{0}} V(x, t ; 0, \tau)[\psi(T)-L] d \tau\right| \leqslant \frac{4 M}{\sqrt{\pi}} \int_{x / 2 \sqrt{t}}^{x / 2 \sqrt{t}-T_{0}} e^{-\sigma^{2}} d \sigma
$$

et on peut choisir un nombre $T_{1}>T_{0}$ de sorte que l'on ait

(21) $\quad\left|\frac{1}{2 V_{\pi}}-\int_{0}^{T_{0}} V(x, t ; 0, \tau)[\psi(\tau)-L] d \tau\right|<\varepsilon / 2$ pour $\quad t>T_{1}$.

On déduit de $(20)$ et de (21) l'inégalité

$$
\left|\frac{1}{2 \sqrt{\pi}}-\int_{0}^{t} V(x, t ; 0, \tau)[\psi(\tau)-L] d \tau\right| \leqslant \varepsilon \quad \text { pour } \quad t>T_{1},
$$

il est done évident que le premier terme du second membre de (18) tend vers zéro pour $t \rightarrow \infty$. Il en résulte que $\lim _{t \rightarrow \infty} J_{1}(x, t)=L$.

CoRoLlaIRE. Soit $u(x, t)$ la solution du premier problème de Fourier avec les conditions (3) et (5), la fonction $\varphi(x)$ étant continue et bornée pour $x \geqslant 0$, tandis que la fonction $\psi(t)$ est continue pour $t \geqslant 0$. Supposons que l'on ait

Alors on a

$$
\lim _{t \rightarrow \infty} \psi(t)=L .
$$

$$
\lim _{t \rightarrow \infty} u(x, t)=L
$$

pour toute valeur fixée, positive de la variable $x$.

\section{Etude de l'intégrale $J_{0}(x, t)$}

9. Avec les mêmes hypothèses sur la fonction $\psi(t)$, le potentiel calorifique $J_{0}(x, t)$, en général, n'a pas de limite pour $t \rightarrow \infty$. Considérons par exemple l'intégrale

$$
j_{0}(x, t)=\frac{1}{2 \sqrt{\pi}} \int_{0}^{t} \frac{1}{(t-\tau)^{1 / 2}} \exp \left[-\frac{x^{2}}{4(t-\tau)}\right] d \tau .
$$

En posant $\tau=t-x^{2} / 4 z^{2}$ et en intégrant par parties, on obtient

$$
\begin{aligned}
j_{0}(x, t) & =\frac{|x|}{2 \sqrt{\pi}} \int_{|x| / 2 \sqrt{t}}^{\infty} z^{-2} \exp \left(-z^{2}\right) d z \\
& =\sqrt{\frac{t}{\pi}} \exp \left(-\frac{x^{2}}{4 t}\right)-\frac{|x|}{\sqrt{\pi}} \int_{|x| / 2 \sqrt{t}}^{\infty} \exp \left(-z^{2}\right) d z .
\end{aligned}
$$

Il résulte de la formule (23) que l'on a

$$
\lim _{t \rightarrow \infty} j_{0}(x, t)=\infty
$$

Examinons encore l'intégrale

$$
j_{a}(x, t)=\frac{1}{2 \sqrt{\pi}} \int_{0}^{t} \frac{1}{(t-\tau)^{1 / 2} \tau^{a}} \exp \left[-\frac{x^{2}}{4(t-\tau)}\right] d \tau \quad(0<a<1),
$$

c'est-à-dire le cas particulier de l'intégrale $J_{0}(x, t)$ correspondant à $\psi(t)$ $=t^{-\alpha}$. Posons $\tau=t z$; l'intégrale $j_{\alpha}$ prend la forme

$$
j_{a}(x, t)=\frac{t^{1 / 2-a}}{2 \sqrt{\pi}} \int_{0}^{1} \frac{d z}{(1-z)^{1 / 2} z^{a}} \exp \left[-\frac{x^{2}}{4 t(1-z)}\right] d z .
$$

L'intégrale qui figure au second membre est convergente pour toute valeur positive de $t$; elle est inférieure à $B\left(\frac{1}{2} ; 1-\alpha\right)$ et il résulte du théorème de Lebesgue, cité au $\mathrm{n}^{\circ} 6$, qu'elle tend vers $B\left(\frac{1}{2} ; 1-\alpha\right)$ pour $t \rightarrow \infty$. Il en résulte que l'on a

$$
\therefore \lim _{t \rightarrow \infty} j_{a}(x, t)=\left\{\begin{array}{cll}
\infty & \text { pour } & \alpha<\frac{1}{2}, \\
B\left(\frac{1}{2}, 1-\alpha\right) / 2 \sqrt{\pi} & \text { pour } \quad \alpha=\frac{1}{2}, \\
0 & \text { pour } \quad \frac{1}{2}<\alpha<1 .
\end{array}\right.
$$

L'étude de l'intégrale $j_{a}(x, t)$ montre que l'intégrale $J_{0}(x, t)$ peut ne pas avoir de limite, mềme lorsque la fonction $\psi(t)$ tend vers zéro pour $t \rightarrow \infty$. Pourtant nous allons démontrer le théorème suivant:

ThÉoRìme. Si la fonotion $\psi(t)$ est continue pour $t \geqslant 0$ et satisfait a l'inégalité
(24)

$$
|\psi(t)| \leqslant M t^{-\alpha},
$$

$M$ étant un nombre positif et a un nombre supérieur $\dot{a} \frac{1}{2}$, on a

$$
\lim _{t \rightarrow \infty} J_{0}(x, t)=0
$$

pour toute valeur non négative de $x$. 
Démonstration. Posons pour abréger

$$
U(x, t ; \xi, \tau)=(t-\tau)^{-1 / 2} \exp \left[-(x-s)^{2} / 4(t-\tau)\right],
$$

et soit $t_{0}$ un nombre positif quelconque. On a

(25) $J_{0}(x, t)=\frac{1}{2 \sqrt{\pi}} \int_{0}^{t} U(x, t ; 0, \tau) \psi(\tau) d \tau$

$$
=\frac{1}{2 \sqrt{\pi}} \int_{0}^{t_{0}} U(x, t ; 0, \tau) \psi(\tau) d \tau+\frac{1}{2 \sqrt{ } \pi} \int_{i_{0}}^{t_{0}} U(x, t ; 0, \tau) \psi(\tau) d \tau .
$$

Il est évident que le premier terme du second membre de (25) tend vers zéro pour $t \rightarrow \infty$. Quant à l'intégrale qui figure an second terme, on a

$$
\left|\int_{t_{0}}^{t} \frac{\psi(\tau)}{(t-\tau)^{1 / 2}} \exp \left[-\frac{x^{2}}{4(t-\tau)}\right] d \tau\right| \leqslant M \int_{t_{0}}^{t} \frac{d \tau}{(t-\tau)^{1 / 2} \tau^{\alpha}} \leqslant M t^{1 / 2-\alpha} B\left(\frac{1}{2}, 1-\alpha\right),
$$

par conséquent, le second terme du second membre de (25) tend aussi vers zéro pour $t \rightarrow \infty$. Ceci achève là démonstration du théorème.

10. L'étude de l'allure asymtotique de l'intégrale $J_{0}$ pour $t \rightarrow \infty$, que nous avons poursuivie au $\mathrm{n}^{\circ} 9$, permet de faire quelques remarques concernant les solutions du second problème de Fourier, avec les conditions (3) et '(9). Observons d'abord que la fonction $u(x, t)=A x$ ( $A$ b́tant un nombre constant) constitue une solution (la seule dans la classe $\boldsymbol{E}_{\mathrm{a}}$ ) de l'équation (1), satisfaisant à la condition initiale $u(x, 0)=A x$ pour $x \geqslant 0$, et à la condition aux limites $u_{x}^{\prime}(0, t)=A$. D'autre part, en appliquant la formule (10), on peut mettre cette solution sous la forme

$$
\begin{aligned}
u(x, t)= & -\frac{\mathrm{A}}{\sqrt{\pi}} \int_{0}^{t} \frac{1}{(t-\tau)^{1 / 2}} \exp \left[-\frac{x^{2}}{4(t-\tau)}\right] d \tau+ \\
& +\frac{A}{2 \sqrt{\pi t}} \int_{0}^{\infty} s\left\{\exp \left[-\frac{(x-s)^{2}}{4 t}\right]+\exp \left[-\frac{(x+s)^{2}}{4 t}\right]\right\} d s,
\end{aligned}
$$

c'est-à-dire

(26)

$$
u(x, t)=-2 A j_{0}(x, t)+A u_{0}(x, t)
$$

(voir $\mathrm{L}^{0} 9$ ), avec

$$
u_{0}(x, t)=\frac{1}{2 \sqrt{\pi t}}\left\{\int_{0}^{\infty} s \exp \left[-\frac{(x-s)^{2}}{4 t}\right] d s+\int_{0}^{\infty} s \exp \left[-\frac{(x+s)^{2}}{4 t}\right] s\right\} .
$$

En posant $s=x+2 \sigma \sqrt{t}$, resp. $s=-x+2 \sigma \sqrt{t}$ dans les intégrales du second membre, on obtient

$$
u_{0}(x, t)=\frac{x}{\sqrt{\pi}} \int_{-x / 2 \sqrt{/} / 2 \sqrt{t}}^{x / \sigma^{2}} d \sigma+2 \sqrt{\frac{t}{\pi}} \exp \left(-\frac{x^{2}}{4 t}\right) .
$$

Si l'on substitue dans (26) les expressions pour $j_{0}(x, t)$ et $u_{0}(x, t)$ d'après les formules (23) et (27), les termes tendant vers l'infini pour $t \rightarrow \infty$, se détruisent, de sorte qu'on aboutit bien à $u(x, t) \equiv A x$.

Soit $u(x, t)$ une fonction de classe $E_{2}$, constituant une solution du second problème de Fourier pour l'équation (1), avec les conditions (3) et $(9)$, représentée par la formule (10). Supposons que les fonctions $\varphi(x)$ et $\psi(t)$ soient mises respectivement sous la forme $\varphi(x)=A x+\varphi_{0}(x)$, $\psi(t)=B+\psi_{0}(t), A$ et $B$ étant des constantes, et $\varphi_{0}(x)$ et $\psi_{0}(t)$ des fonctions continues pour $x \geqslant 0$ et $t \geqslant 0$ respectivement. Nous supposons en outre que l'on a

$$
\lim _{x \rightarrow \infty} \varphi_{0}(x)=l,
$$

tandis que la fonction $\psi(t)$ satisfait à la condition (24) avec $a>\frac{1}{2}$. Soit $\varphi^{\prime}(0)=\psi(0)$. Il résulte de nos considérations que si l'on a $A=B$, la fonction $u(x, t)$ représentée par la formule $(10)$ tend vers la limite $l$ pour $t \rightarrow \infty$. Si au contraire $A \neq B$, la fonction $u(x, t)$ n'a pas de limite pour $t \rightarrow \infty$.

\section{Travaux cités}

[1] E. Goursat, Oours d'analyse mathématique, vol. III, Paris 1927.

[2] C. de la Valléo-Poussin, Intégrales de Lebesgue, Paris 1916.

[3] А. Н. Тихонов и А. А. Самарс ки й, Уравнения математической физики, Москва 1951. 